Received: 31 January 2018

Accepted: 28 January 2019

Published online: 27 February 2019

NTIFIC REP

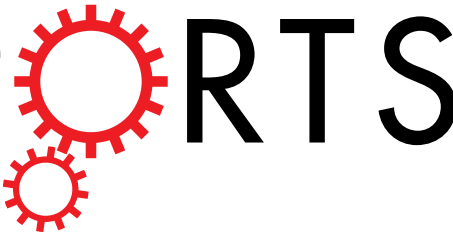

\title{
OPEN A familial congenital heart disease with a possible multigenic origin involving a mutation in BMPR1A
}

Till Joscha Demal ${ }^{1,2}$, Melina Heise ${ }^{3}$, Benedikt Reiz ${ }^{1}$, Deepika Dogra ${ }^{4}$, Ingrid Brænne ${ }^{1,5}$, Hermann Reichenspurner ${ }^{2}$, Jörg Männer ${ }^{6}$, Zouhair Aherrahrou ${ }^{1}$, Heribert Schunkert ${ }^{7}$, Jeanette Erdmann ${ }^{1}$ \& Salim Abdelilah-Seyfried ${ }^{3,4}$

The genetics of many congenital heart diseases (CHDs) can only unsatisfactorily be explained by known chromosomal or Mendelian syndromes. Here, we present sequencing data of a family with a potentially multigenic origin of CHD. Twelve of nineteen family members carry a familial mutation [NM_004329.2:c.1328G $>$ A (p.R443H)] which encodes a predicted deleterious variant of BMPR1A. This mutation co-segregates with a linkage region on chromosome 1 that associates with the emergence of severe CHDs including Ebstein's anomaly, atrioventricular septal defect, and others. We show that the continuous overexpression of the zebrafish homologous mutation bmpr1aa ${ }^{p . R 438 H}$ within endocardium causes a reduced $A V$ valve area, a downregulation of $W n t / B$-catenin signalling at the $A V$ canal, and growth of additional tissue mass in adult zebrafish hearts. This finding opens the possibility of testing genetic interactions between BMPR1A and other candidate genes within linkage region 1 which may provide a first step towards unravelling more complex genetic patterns in cardiovascular disease aetiology.

Congenital heart diseases (CHDs) are the most common organ malformations and affect $1 \%$ of newborns ${ }^{1,2}$. Due to recent improvements in the treatment of CHDs, increasing numbers of patients reach a reproductive age. This has raised renewed interest in understanding the molecular causes of CHDs with the aim of improving diagnostic or therapeutic tools. Although a variety of genes has been implicated in the development of CHDs, only a minority of these diseases is caused by monogenic mutations ${ }^{3}$. Hence, one of the most urgent challenges in cardiovascular disease aetiology is a better understanding of more complex genetic traits leading to CHDs.

A large proportion of all CHDs affect the formation of atrioventricular (AV) valves. In higher vertebrates, the endocardial cushions are precursors of AV valves, cardiac septa, and parts of the cardiac outflow tract. The atrioventricular endocardial cushions are formed by endocardial cells of the atrioventricular canal (AVC) that hypertrophy and migrate into the extracellular matrix in between the inner endocardial and the outer myocardial layer of the heart tube ${ }^{4}$. This process is known as endothelial-mesenchymal transition (endoMT). Afterwards the endocardial cushions located in the AVC form the atrioventricular valves ${ }^{5}$. Defective development of the endocardial cushions can lead to CHDs including atrial, ventricular, and atrioventricular septal defects in mice ${ }^{6,7}$.

The zebrafish is an excellent vertebrate model for functional studies of valve leaflet morphogenesis ${ }^{8}$. The zebrafish and human genome share a high degree of similarity with $69 \%$ of protein-coding zebrafish genes being related to genes found in humans ${ }^{9}$. Hence, the analysis of human congenital defects is feasible in this animal model. In contrast to human anatomy, the zebrafish heart consists of only one atrium and ventricle. These two

${ }^{1}$ Institute for Cardiogenetics, University Heart Centre Lübeck, University of Lübeck, DZHK (German Research Centre for Cardiovascular Research) partner site Hamburg/Lübeck/Kiel, D-23562, Lübeck, Germany. ${ }^{2}$ Department of Cardiovascular Surgery, University Heart Centre Hamburg, D-20246, Hamburg, Germany. ${ }^{3}$ Institute of Molecular Biology, Hannover Medical School, D-30625, Hannover, Germany. ${ }^{4}$ Institute of Biochemistry and Biology, Potsdam University, D-14476, Potsdam, Germany. ${ }^{5}$ Center for Public Health Genomics, University of Virginia, Charlottesville, VA, 22903, USA. ${ }^{6}$ Institute of Anatomy and Embryology, UMG, Göttingen University, D-37075, Göttingen, Germany. ${ }^{7}$ Department of Cardiovascular Diseases, German Heart Centre Munich, Technical University of Munich (TUM) and DZHK (German Research Centre for Cardiovascular Research) partner site, D-80636, Munich, Germany. Till Joscha Demal, Melina Heise, Jeanette Erdmann, and Salim Abdelilah-Seyfried contributed equally. Correspondence and requests for materials should be addressed to J.E. (email: jeanette.erdmann@uni-luebeck.de) or S.A.-S. (email: salim.seyfried@uni-potsdam.de) 


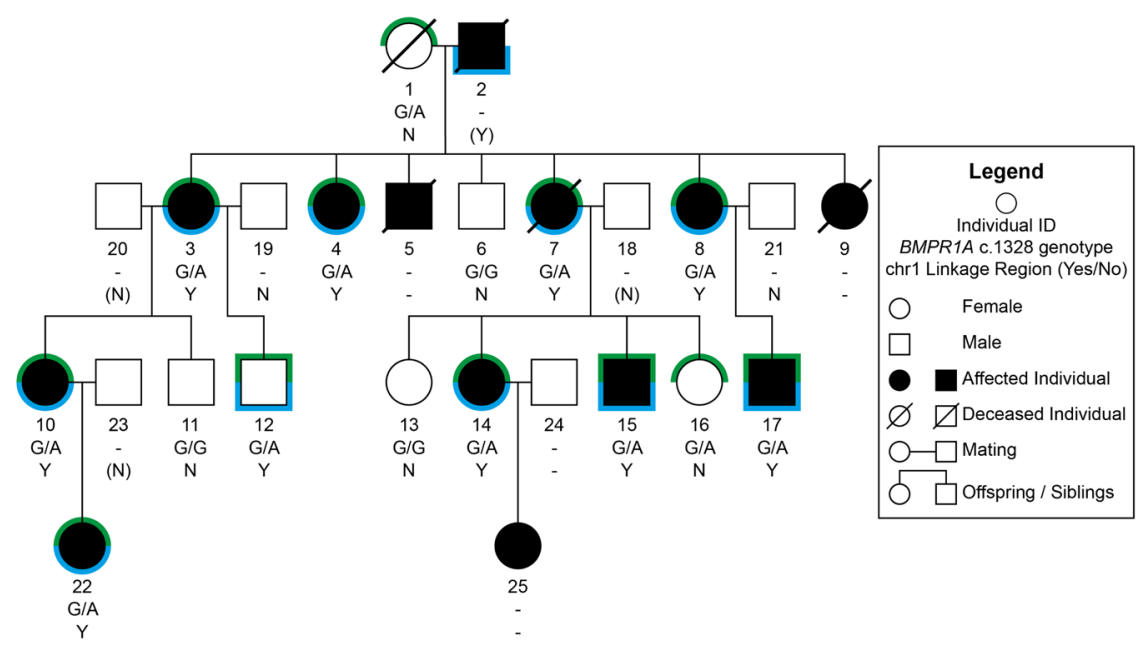

Figure 1. Pedigree of the affected family. Green marks indicate that the individual carries the BMPR1A mutation and blue marks represent the co-segregation of the chromosome 1 linkage region. For each individual, personal ID, $B M P R 1 A^{c .1328}$ genotype, and co-segregation status of the chromosome 1 linkage region is listed. G/A: Heterozygote carrier of $B M P R 1 A^{p . R 443 H}$. G/G: No mutation. '-': No sequencing data available. N: No; Y: Yes. Values in brackets are based on the haplotype.

cardiac chambers are separated by an AV valve. During zebrafish cardiac valve development, cardiac cushions elongate and form paired primitive bicuspid valve leaflets, which protrude from either side of the AVC into the lumen ${ }^{10,11}$. Within three months, the initially bicuspid valves transform into quadricuspid structures ${ }^{12}$.

The bone morphogenetic protein (BMP) pathway plays an important role in the development of embryonic heart valves ${ }^{7,13,14}$. BMPs are involved in the development of endocardial cushions via endoMT, the maturation of the tissue surrounding the AV valves, and the septation of heart cavities ${ }^{14}$. In mice, expression of BMP-Receptor $1 A$ (BMPR1A, also known as $A L K 3$ ) is required in both endocardium and myocardium to ensure the correct development of endocardial cushions ${ }^{15,16}$.

There are numerous case reports about patients with BMPR1A mutations and cardiac septal defects. These defects often occur in the context of deletion syndromes and are in combination with mental retardation, facial dysmorphism, or juvenile polyposis syndrome (JPS) ${ }^{17-20}$. In addition, isolated BMPR1A mutations have been reported to associate with cardiac malformations and occurrence of JPS. Several missense mutations of BMPR1A are associated with the emergence of ventricular septal defects and Ebstein's anomaly ${ }^{21}$. Mutations in the BMP pathway have also been connected to non-syndromic CHDs. D'Allessandro et al. described three rare mutations of BMPR1A (p.R478H, p.D429V, and p.P481S) and the concomitant occurrence of atrioventricular septal defects $^{22}$.

The involvement of BMPR1A in the development of Ebstein's anomaly has also been shown in animal studies. Mice with a conditional knockout of BMPR1A in the AV canal displayed a malformation of the tricuspid valve and a disruption of the annulus fibrosus with a consecutive ventricular preexcitation, both which are characteristics of Ebstein's anomaly ${ }^{23}$.

Although numerous reports of patients with BMPR1A mutations and associated CHDs exist, a clear causal connection has not yet been demonstrated in functional studies. Since chromosomal and Mendelian syndromes explain only $20 \%$ of the cases ${ }^{24}$, also more complex genetic processes may have an important influence on the development of CHD.

In 1997, we described a family with multiple cardiac cushion defects (e.g. Ebstein's anomaly, atrioventricular septal defect, and aortic stenosis ${ }^{25}$. Within four generations, at least 13 family members were affected. Here we present the results of next generation sequencing of this family. Using an adult zebrafish model, we provide a detailed functional analysis of a candidate mutation in BMPR1A. Our results indicate a more complex genetic trait involved in CHD.

\section{Results}

Phenotype and genotype data of a large pedigree with CHDs. Since the first description by Schunkert et al. of a family as "a large pedigree with valvuloseptal defects ${ }^{25}$ ", two more family members have been identified with 13 of 19 family members suffering from CHDs (Fig. 1). No extracardiac anomalies were reported, apart from a single patient that anamnestically suffered from "severe malformations" before he died during the first days after birth (no medical records available). The main phenotypes were characterized by atrioventricular septal defects (AVSD) $(n=3)$, an atrial septal defect (ASD) $(n=1)$, a ventricular septal defect (VSD) $(n=1)$, Ebstein's anomalies $(n=4)$, Wolff-Parkinson-White (WPW) syndromes $(n=3)$, cleft mitral valves $(n=3)$, and right bundle branch blocks (RBBB) $(n=3)$ (see Supplementary Table S1 for a summary of all available clinical features and patient details).

The family was first characterized by short tandem repeat (STR)-based linkage analysis ${ }^{26}$. Although this led to the discovery of a linked region on chromosome 1, the signal did not yield genome-wide significance based 

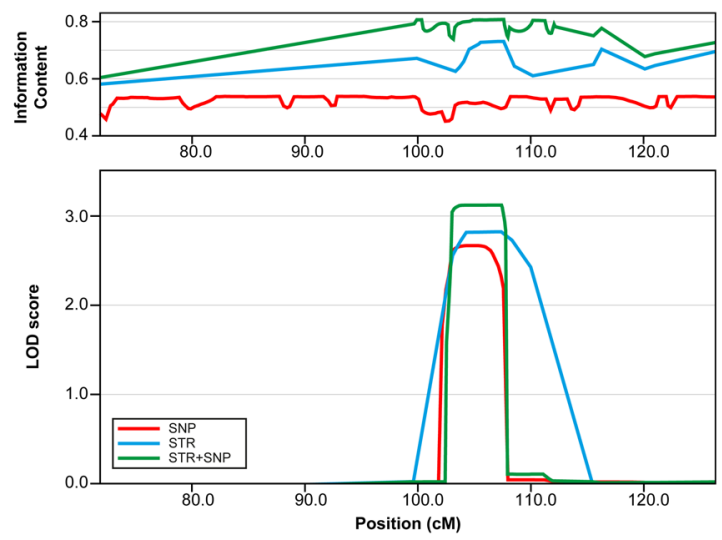

Figure 2. Chromosome 1 linkage region based on SNP and STR data. SNP data (red): information content $\sim 0.5$, peak LOD score 2.663 at position $104.823-105.289 \mathrm{cM}$ (80675529-81258943 bp); STR data (blue): information content $\sim 0.6-0.7$, peak LOD score 2.817 at position $106.85-107.4 \mathrm{cM}(81998598-82895464 \mathrm{bp})$. Combination of SNP and STR data (green): information content $\sim 0.6-0.8$, peak LOD score 3.118 at position 106.666-107.4cM (81911788-82895464bp).

on an established logarithm of the odds (LOD)-score threshold of 3. Given the large region and the limitations in sequencing technologies at that time, the analysis rested until, almost two decades later, we reanalysed the family by current state-of-the-art linkage analysis techniques combined with genome and exome sequencing.

We combined the STR data from candidate linkage regions with dense SNP markers analysis. STR data was available for individuals $1,3,4,6-8,10,11,13-17,19$, and 21 . SNP data was available for the individuals 3,4 , $8,10-13,15,17$, and 22 (Fig. 1). In total, five distinct linkage signals on chromosomes 1, 2, 4, 10, and X were detected by a nonparametric linkage analysis (Supplementary Fig. S1). Based on the combined STR and SNP data, the only signal which exceeded a LOD score of 3 was on chromosome 1 (Fig. 2). The LOD score was above 3 at genomic position hg19 chr1: 77887835-83041391 bp and overlaps with the RefSeq genes ADGRL2, ADGRL4, AK5, DNAJB4, FUBP1, GIPC2, IFI44, IFI44L, LINC01781, LOC101927412, LOC101927434, MGC27382, MIGA1, NEXN, PTGFR, USP33, and ZZZ3.

All of the $10 \mathrm{CHD}$ affected family members that were available for sequencing analysis carry that linkage region (Fig. 1). The odds ratio $(\mathrm{OR})$ is significantly higher than $1(\mathrm{p}=0.0001$, Fisher's exact test). One unaffected family member (Individual No. 12) also carries the linkage region, which suggests reduced penetrance, a multifactorial inheritance, and/or the contribution of environmental factors.

After the identification of potential disease-associated loci by linkage analysis, we screened the chromosome 1 region in more detail with the aim to identify candidates for disease-causing variants. However, a clear pathogenic variant was not identified within the coding regions of genes in the linkage region on chromosome 1 . Due to limited DNA availability, not all family members were sequenced.

One variant that strongly co-segregated with linkage region 1 was within the BMPR1A locus on chromosome 10 (hg19 genomic position: chr10:g.88681438 G > A). This variant causes a predicted deleterious AA-change [NM_004329.2:c.1328 G > A(p.(R443H)] as indicated by multiple functional prediction tools including SIFT $^{27}$, PolyPhen $2^{28}$, MutationTaster2 ${ }^{29}, \mathrm{CADD}^{30}$, and DANN ${ }^{31}$. So far, this variant has only been reported in the ClinVar registry describing an association to the Hereditary cancer-predisposing syndrome ${ }^{32}$. Subsequent validation of this variant in other family members revealed, that the co-segregation is not perfect: although all affected family members carried the variant, it was also present in three unaffected family members (Fig. 1; No. 1, 12, and 16). However, the variant occurs significantly more frequently in family members suffering from CHD when compared to their unaffected relatives $(\mathrm{p}=0.044$, Fisher's exact test).

Because BMPR1A $A^{p . R 43 H}$ (chromosome 10) and the linkage region on chromosome 1 showed a strong co-segregation within the family, we next tested whether a chromosomal translocation was present. However, this possibility was excluded by Fluorescence in situ hybridization (FISH) analysis.

The human BMPR1A ${ }^{p . R 443 H}$ mutation complements the loss of zebrafish Bmpr1aa/Bmpr1ab receptors and hence encodes a functional receptor variant. To test whether the human gene encoding BMPR1 $\mathrm{A}^{\mathrm{p} \cdot \mathrm{R} 443 \mathrm{H}}$ is functional, we performed a complementation assay based on mRNA injections into zebrafish bmprlaa/ab double morphants. Loss of the two Bmprla proteins in zebrafish causes severe dorsalization defects that can partially be rescued by the injection of human $B M P R 1 A^{W T} \mathrm{mRNA}^{33}$. In addition to using human $B M P R 1 A^{W T}$ and $B M P R 1 A^{p \cdot R 443 H}$ mRNAs, we also injected BMPR1 $A^{p . L 342 R}$ ("Linkspoot") mRNA that contains a missense mutation which produces a dominant-negative BMPR $1 \mathrm{~A}^{34}$. Finally, we also tested $B M P R 1 A^{p . R 443 C}$ that harbours a mutation, which is associated with the occurrence of juvenile polyposis syndrome (JPS) ${ }^{35}$. As $B M P R 1 A^{p . R 443 C}$ (referred to as JPS variant) affects the same residue as the mutation found in the reported family, we used this mutant variant to elucidate possible residue-specific effects on the BMPR1A mutant protein (see Supplementary Table S2 for a list of all BMPR1A variants used in this study; Supplementary Fig. S2 shows a sequence alignment of human BMPR1A with zebrafish Bmprlaa). 

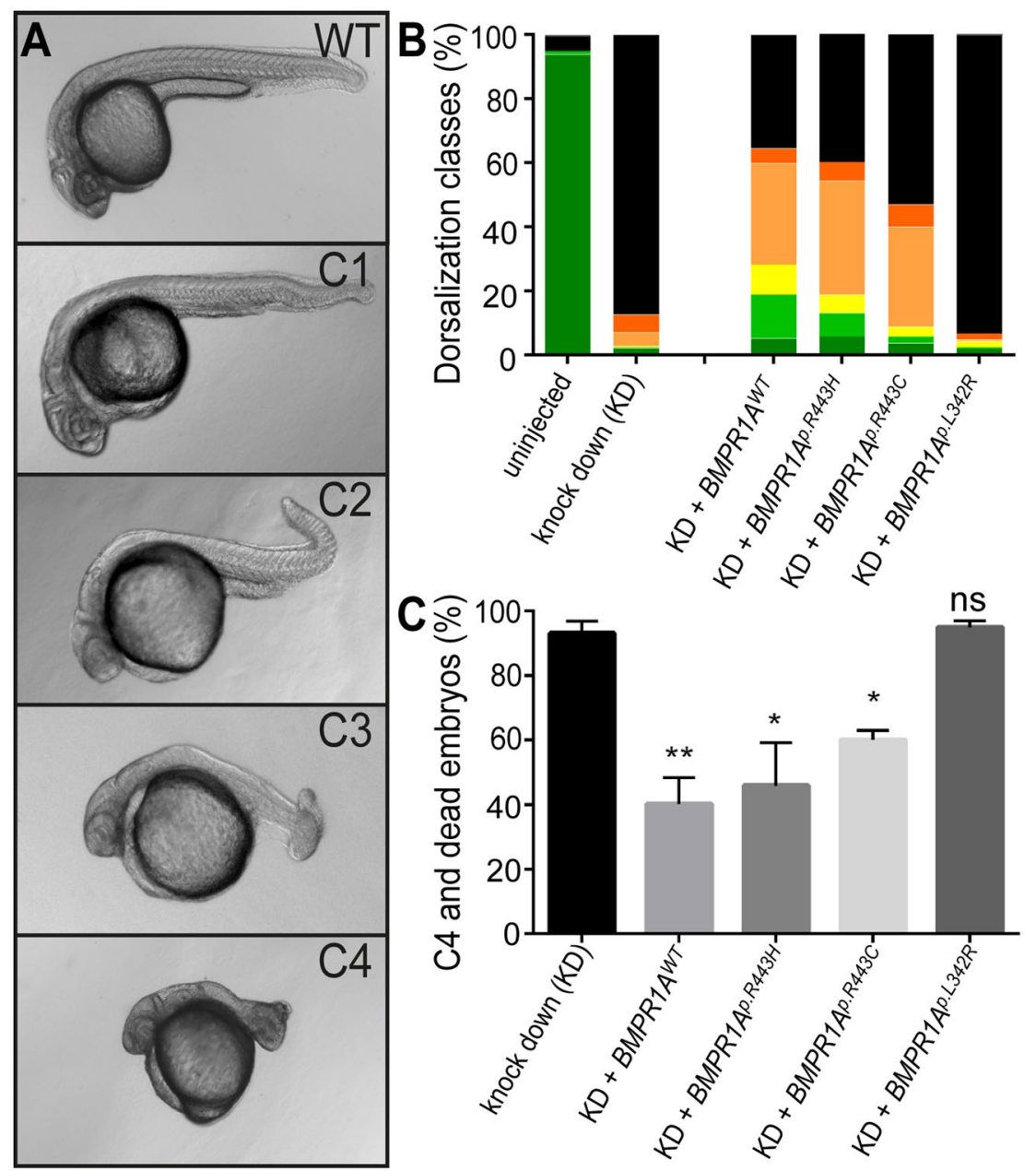

Figure 3. Rescue of bmprlaa morphant dorsalization phenotypes in zebrafish by injection of mRNA encoding human BMPR1A. (A) Representative classes of dorsalization phenotypes in zebrafish embryos at $24 \mathrm{hpf}$ as previously described ${ }^{36}$. (B) Mean percentages of the five dorsalization classes among the different experimental groups [total number of embryos analysed: uninjected, $\mathrm{n}=528$; bmprlaa MO knockdown (KD), $\mathrm{n}=217$; $\mathrm{KD}+B M P R 1 A^{W T}$ mRNA, $\mathrm{n}=228 ; \mathrm{KD}+B M P R 1 A^{p . R 443 H}$ mRNA, $\mathrm{n}=255 ; \mathrm{KD}+B M P R 1 A^{p . R 443 C}$ mRNA (JPS), $\mathrm{n}=147 ; \mathrm{KD}+B M P R 1 A^{p . L 342 R}$ mRNA (Linkspoot), $\left.\mathrm{n}=219\right]$. (C) Share of embryos with C4 phenotype or lethality (mean \pm SEM) after knockdown and human BMPR1A mRNA rescue injections by $24 \mathrm{hpf}$ [Total number of injected embryos: $\mathrm{n}=1066$ (Supplementary Tables S6, S7, S8, S9, S10). Number of experiments: KD, $\mathrm{n}=5 ; \mathrm{KD}+B M P R 1 A^{W T}$ mRNA, $\mathrm{n}=6 ; \mathrm{KD}+B M P R 1 A^{p \cdot R 443 H}$ mRNA, $\mathrm{n}=5 ; \mathrm{KD}+B M P R 1 A^{p \cdot R 443 C}$ mRNA (JPS), $\mathrm{n}=4 ; \mathrm{KD}+B M P R 1 A^{p . L 342 R}$ mRNA (Linkspoot), $\mathrm{n}=4$. Indicated is the statistical significance of the difference between the occurrence of severe dorzalisation defects in each condition compared with the pure knockdown (KD) $(* \mathrm{p} ;<0.05, * * \mathrm{p}<0.01$, ns: not significant $)]$.

For complementation assays, we co-injected BMPR1A mRNAs together with antisense oligonucleotide morpholinos (MO) against bmprlaa and bmpr1ab. At 24hpf, zebrafish embryos were classified into one of four different dorsalization classes that were categorized from $\mathrm{C} 1$ to $\mathrm{C} 4$ with ascending severity as previously described ${ }^{36}$ (Fig. 3A).

As previously shown, co-injection of BMPR1A p.L342R ("Linkspoot") mRNA together with bmpr1aa/ab MOs did not rescue the morphant phenotype ${ }^{34}$. In comparison, all other mRNAs showed a statistically significant rescue (Fig. 3C). Hence, contrary to our expectations based on the functional prediction tools used in this study, human BMPR1 $\mathrm{A}^{\mathrm{p} . \mathrm{R} 443 \mathrm{H}}$ and BMPR1 $\mathrm{A}^{\mathrm{p} \cdot \mathrm{R} 443 \mathrm{C}}$ (JPS variant) receptor variants are functional in zebrafish and functionally complement the Bmprlaa/ab knockdown-associated dorsalization phenotypes in zebrafish embryos. This finding provided further evidence for a genetic pattern of inheritance associated with the CHDs that is more complex than initially assumed.

The diameter of zebrafish embryonic $\mathrm{AV}$ valves is not affected by pan-endothelial/endocardial overexpression of a bmpr1aa ${ }^{p . R 438 H}$ mutant. Bmp signalling has an essential role during valve development in mice, where it controls endoMT ${ }^{16}$. In zebrafish, $b m p 4$ is highly expressed at the cardiac cushions and Bmp receptors including Bmprlaa are expressed within the early endocardium ${ }^{37}$. To elucidate 

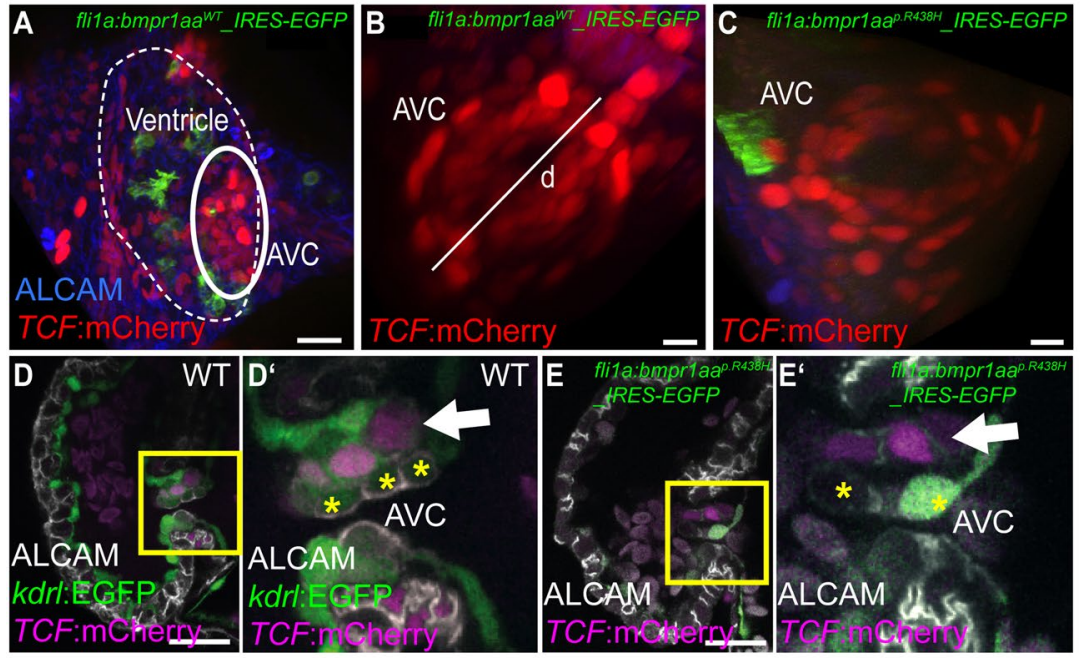

Figure 4. Pan-endothelial expression of a zebrafish Bmprlaa ${ }^{\mathrm{p} \cdot \mathrm{R} 438 \mathrm{H}}$ mutant protein does not affect embryonic valvulogenesis. (A-E) Shown are reconstructions of confocal z-stack images of the zebrafish embryonic atrioventricular canal (AVC) region. (A) Embryonic cardiac ventricle in a $T g(f l i 1 a: G a l 4 F F)^{u b s 3}$; Tg(UAS:bmpr1aa WT_IRES_EGFP) ${ }^{m d 65}$; Tg(7xTCF-Xia.Sia:NLS-mCherry) ${ }^{i a 5}$ zebrafish at $120 \mathrm{hpf}$. The AVC region is outlined. Scale bar $=20 \mu \mathrm{m}$. (B) AVC region in a Tg(fli1a:Gal4FF $)^{u b s 3}$; Tg(UAS:bmpr1aa ${ }^{W T}$ IRES_EGFP $)^{\text {md65; }}$; Tg(7xTCF-Xia.Sia:NLS-mCherry $)^{i a 5}$ or (C) Tg(fli1a:Gal4FF $)^{u b s 3}$; Tg(UAS:bmpr1aa ${ }^{p \cdot R 438 H}{ }_{\text {IRES_EGFP }}{ }^{m d 60}$; Tg(7xTCF-Xia.Sia:NLS-mCherry) ${ }^{i a 5}$ zebrafish at $120 \mathrm{hpf}$. Indicated is the diameter of the AVC (d). Scale bar $=10 \mu \mathrm{m} .\left(\mathbf{D}, \mathbf{D}^{\prime}\right)$ Embryonic valve formation in the WT zebrafish embryo at $72 \mathrm{hpf}$. Box indicates the region shown in $\left(\mathbf{D}^{\prime}\right)$. Valve leaflets in $\mathrm{WT}$ embryos are characterized by double-layering with an abluminal population of AVC cells that has active Wnt signalling marked by $\mathrm{Tg}(7 x \mathrm{TCF}$-Xia.Sia:NLS-mCherry) (arrow). Cell membranes of luminal cells are marked by immuno-labelling against ALCAM (cells marked by asterisks). $\left(\mathbf{E}, \mathbf{E}^{\prime}\right)$ Similarly, valvulogenesis is not affected in $\mathrm{Tg}(\text { fli1a:Gal4FF })^{\text {ubs }} ; \mathrm{Tg}\left(\mathrm{UAS}: b m p r 1 a a^{p . R 438 H} \text { IRES_EGFP }\right)^{\text {md60 }}$; $\operatorname{Tg}$ (7xTCF-Xia.Sia:NLS-mCherry) ${ }^{i a}$ embryos that show a normal double-layered leaflet morphology with Wnt signalling in abluminal cells (arrow) and ALCAM-positive luminal cells (asterisks). Scale bars $=20 \mu \mathrm{m}$. (F) The diameter of the AVC in zebrafish embryos with pan-endothelial overexpression of bmprlaa ${ }^{p . R 438 H}$ does not significantly differ from that upon bmprlaa ${ }^{W T}$ overexpression [total number of embryos analysed: $b m p r 1 a a^{W T}$, $\mathrm{n}=6 ;$ mprlaa ${ }^{p \cdot R 438 H}, \mathrm{n}=8$; two-sided student's t-test, $\left.\mathrm{p}=0,066\right]$.

whether a zebrafish Bmprlaa ${ }^{\text {p.R438H }}$ variant corresponding to the human BMPR1A $A^{\text {p.R443H }}$ variant exerts some dominant-negative or gain-of function activity which may not be detected during dorsoventral pattern formation of the zebrafish embryo, we next analysed its activity during zebrafish cardiac valve leaflet formation. To this end, we generated two stable transgenic lines of zebrafish for Gal4-dependent overexpression of bmprlaa ${ }^{p . R 438 H}$ $\left[T g\left(U A S: b m p r 1 a a^{p . R 438 H}\right.\right.$ IRES_EGFP)] or bmprlaa ${ }^{W T}$ variants [Tg(UAS:bmpr1aa ${ }^{W T}$ IRES_EGFP)]. We used these stable transgenic lines in combination with the pan-endothelial activator line $\bar{T} g(f l i 1 \bar{a}: G A L 4 F F)^{u b s 338}$ to drive expression specifically within endocardium/endothelium. We validated that expression from these transgenic overexpression lines was detectable within endothelium, by performing whole mount in situ hybridizations using an EGFP probe against the bicistronic bmpr1aa_IRES_EGFP mRNAs (Supplementary Fig. S3).

Functional studies of early cardiac development revealed that three independent stable transgenic lines carrying the mutation (allele numbers md60, md61, and md66) were phenotypically not distinguishable. Similarly, two stable transgenic lines with the WT version of the receptor gene (allele numbers md65 and md67) did not show any phenotypes.

In functional tests, we compared valve size and morphology in $b m p r 1 a a^{p \cdot R 438 H}$ or $b m p r 1 a a^{W T}$-overexpressing zebrafish embryos at $120 \mathrm{hpf}$. At that stage, the AVC diameter was marked by the transgenic Wnt signalling

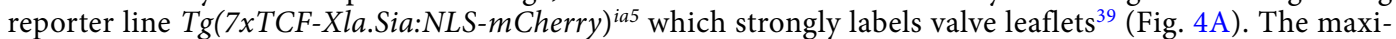
mum AVC diameter was measured from edge to edge of the labelled valve leaflets (Fig. 4B). However, the AVC diameters of embryos expressing either of the two bmprlaa variants did not significantly differ (bmprlaa ${ }^{p . R 438 H}$ : $60.2 \pm 15.1 \mu \mathrm{m}, \mathrm{n}=8 ;$ bmprlaa ${ }^{W T}: 74.1 \pm 8.4 \mu \mathrm{m}, \mathrm{n}=6$; two-sided student's t-test, $\mathrm{p}=0.066$, Fig. $\left.4 \mathrm{~F}\right)$. Obvious morphological changes due to the overexpression of bmpr1aa ${ }^{p . R 438 H}$ were not detectable (Fig. 4D,E). Hence, the endocardial/endothelial expression of $b m p r l a a^{p . R 438 H}$ does not obviously interfere with growth or morphology of zebrafish AV valves during embryogenesis.

Adult zebrafish expressing the $b m p r 1 a a^{p . R 438 H}$ variant have smaller AV valves and reduced Wnt signalling within adult valve leaflets. During growth of zebrafish heart, the initially bicuspid AV valves undergo some remodelling to form the mature quadricuspid valves ${ }^{12,40}$. However, the precise timing and mechanism of this remodelling process is still unknown. To analyse the potential impact of the mutant Bmprlaa variant on this valve remodelling process, we raised zebrafish to adulthood that were continuously expressing either bmprlaa ${ }^{p . R 438 H}$ or bmprlaa ${ }^{W T}$ within endocardial/endothelial cells. We also employed the transgenic Wnt reporter 

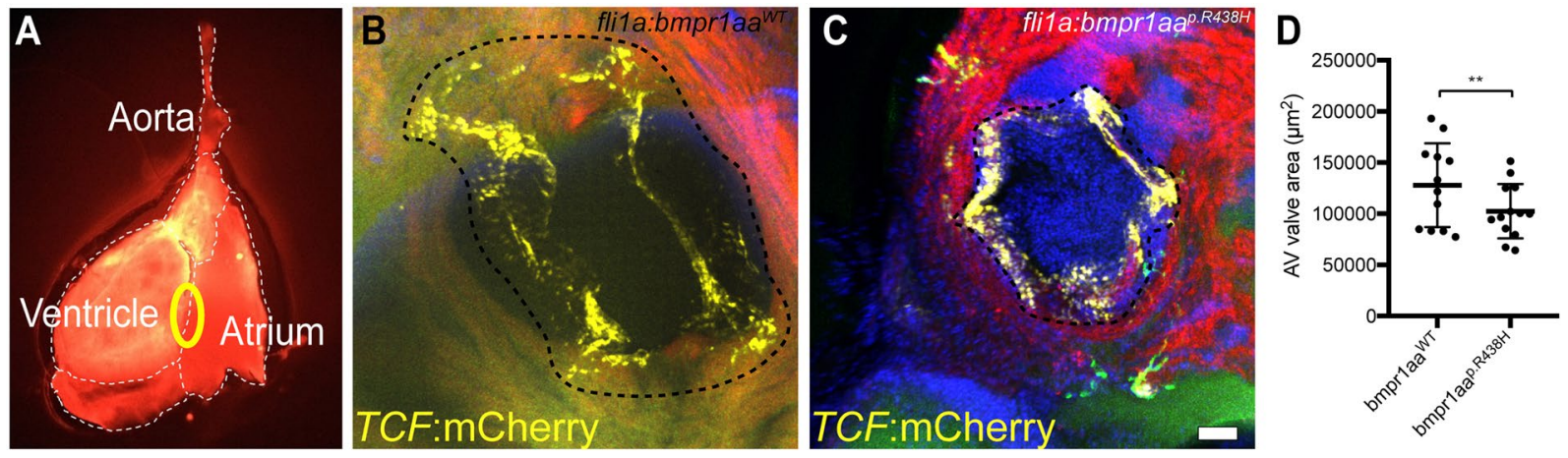

Figure 5. Adult zebrafish with pan-endothelial expression of Bmprlaa ${ }^{\text {p.R } 438 \mathrm{H}}$ have smaller $\mathrm{AV}$ valves. (A) Fluorescence microscopy image of an adult zebrafish heart (ventral view). Circle indicates the position of the AV valve. (B,C) Confocal $\mathrm{z}$-stack maximum intensity projection of adult AV valves of zebrafish with (B) $\mathrm{Tg}(f l i 1 a: G a l 4 F F)^{u b s 3}$; $\mathrm{Tg}\left(\text { UAS:bmpr1aa }{ }^{W T} \text { IRES_EGFP }\right)^{\text {md65; }}$ Tg(7xTCF-Xia.Sia:NLS-mCherry $)^{i a 5}$ or (C)

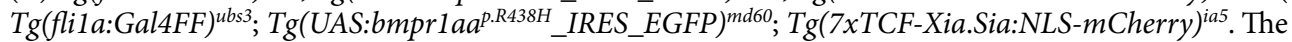
interrupted line indicates the AV valve annulus. This valve annulus surrounds the edge of the valve leaflets marked by TCF expression and occurs in the confocal images as a black ring. The area bounded by the AV valve annulus was measured to quantify the valve size. The AV valve measurement technique is further explained in Supplementary Fig. S4. Scale bar $=50 \mu \mathrm{m}$. (D) Scatter plot of the AV valve area measurements. Adult zebrafish overexpressing Bmprlaa ${ }^{\mathrm{p} . \mathrm{R} 438 \mathrm{H}}$ show a significant reduction in AV valve area [total number of embryos analysed: bmpr1aa ${ }^{W T}, \mathrm{n}=12 ;$ bmpr1aa ${ }^{p . R 438 H}, \mathrm{n}=13$; ANCOVA, $\left.\mathrm{F}(1,22)=10.73 ; * * \mathrm{p}=0.003\right]$.

line $\mathrm{Tg}(7 x \text { TCF-Xla.Sia:NLS- } m \text { Cherry })^{i a 5}$ which strongly labels valve leaflets to identify these structures within the adult heart ${ }^{12,39}$. After raising zebrafish for a period of 3-5 months under conditions of continuous endocardial/ endothelial overexpression of bmpr1aa $a^{p \cdot R 438}$ or $b m p r 1 a a^{W T}$, the AV valvular morphology consisting of four valve leaflets was not affected. Hence, the basic transition from bicuspid to quadricuspid valves occurred normally. To characterize the AV valve morphology in more detail, we extracted transgenic zebrafish hearts and carefully severed the atrium from the ventricle which exposes the AV valve (Fig. 5A). Quantifications of 3D confocal Z-stacks of adult zebrafish AV valves revealed, that the area bounded by the AV valve annulus (Fig. 5B,C) was significantly reduced in animals with a continuous endothelial-specific overexpression of the bmprlaa ${ }^{p . R 438 H}$ mutant when compared to the control fish that were overexpressing bmprlaa ${ }^{W T}\left(b_{m p r l a a^{p . R 438 H}}: 94,638.6 \mu \mathrm{m}^{2}, \mathrm{n}=13\right.$; bmprlaa ${ }^{W T}: 136,352.7 \mu^{2}, \mathrm{n}=12$; ANCOVA; $\mathrm{F}(1,22)=10.73, \mathrm{p}=0.003$; Fig. 5D; Supplementary Fig. S4). Since valve morphogenesis is affected by the size of the developing larvae ${ }^{41}$, an analysis of covariance (ANCOVA) was used, which statistically removes the effects of fish length on AV valve area using linear regression before performing a standard ANOVA. The AV valve measurement technique is further explained in Supplementary Fig. S4.

Canonical Wnt signalling acts as a mitogenic trigger of cushion mesenchyme proliferation following endoMT ${ }^{42}$ and the inhibition of Wnt/ $\beta$-catenin signalling induces a lack of endocardial cushion tissue ${ }^{43}$. During zebrafish embryonic cardiac valve leaflet morphogenesis, the Wnt reporter is expressed on the abluminal side of the forming valve leaflet ${ }^{10,11}$. Strikingly, we found that adult fish overexpressing $b m p r 1 a a^{p . R 438 H}$ within endocardium displayed a severe reduction of $T g(7 x T C F-X l a \text {.Sia:NLS- } m \text { Cherry })^{i a 5}$ reporter expression in comparison to fish overexpressing bmprlaa ${ }^{W T}$ (Supplementary Fig. S4). This finding is indicative of a decreased Wnt signalling activity within adult AV valves upon $b m p r l a a^{p . R 438 H}$ overexpression. Taken together, these findings demonstrate that the pan-endothelial overexpression of $b m p r 1 a a^{p . R 438 H}$ causes a reduced $\mathrm{AV}$ valve size in adult zebrafish that may be triggered by a downregulation of $\mathrm{Wnt} / \beta$-catenin signalling.

Ectopic valvular tissue mass occurs in adult zebrafish with an endocardial overexpression of the bmprlaa ${ }^{p . R 438 H}$ variant. To characterize AV valve morphology in adult zebrafish with an endocardial overexpression of the $b m p r 1 a a^{p . R 438 H}$ variant, we performed an electron-microscopic analysis. We found that three of eight analysed hearts had some growth of ectopic tissue mass on AV valve leaflets (Fig. 6). In comparison, none of seven zebrafish hearts overexpressing $b m p r 1 a a^{W T}$ displayed any such tissue growth ( $\mathrm{p}=0.200$, fisher's exact test). The ectopic tissue growths formed oblong or circular shapes in a size range of $75-150 \mu \mathrm{m}$ in length. This finding provides further evidence for an effect of the bmprla $a^{p . R 43 H}$ variant on cardiac morphology.

\section{Discussion}

As the genetics of most CHDs is unsatisfactorily explained by monogenic inheritance, more complex inheritance patterns gain importance. Here, we report a family with an exceptionally high percentage of affected members (68\%, or 13 out of 19$)$ and a potentially multigenic inheritance of CHDs. Every affected family member for which complete sequencing data is available carries both a BMPR1A missense mutation and a defined linkage region on chromosome 1 (Fig. 1). Therefore, an interaction of the BMPR1A mutation with another genetic entity within the linkage region on Chr.1 may be causative for the diseases.

BMPR1A plays a crucial role during gastrulation and differentiation of mesodermal cells ${ }^{33}$. As Bmprla knockout mice die by day 10 of development ${ }^{44}$, a complete loss of BMPR1A would presumably result in human neonatal death as well. Since embryonic development is not affected in the presented family, it appears unlikely that the BMPR1A ${ }^{p . R 43 H}$ mutation causes a complete loss-of-function of BMPR1A signalling. This is in agreement 

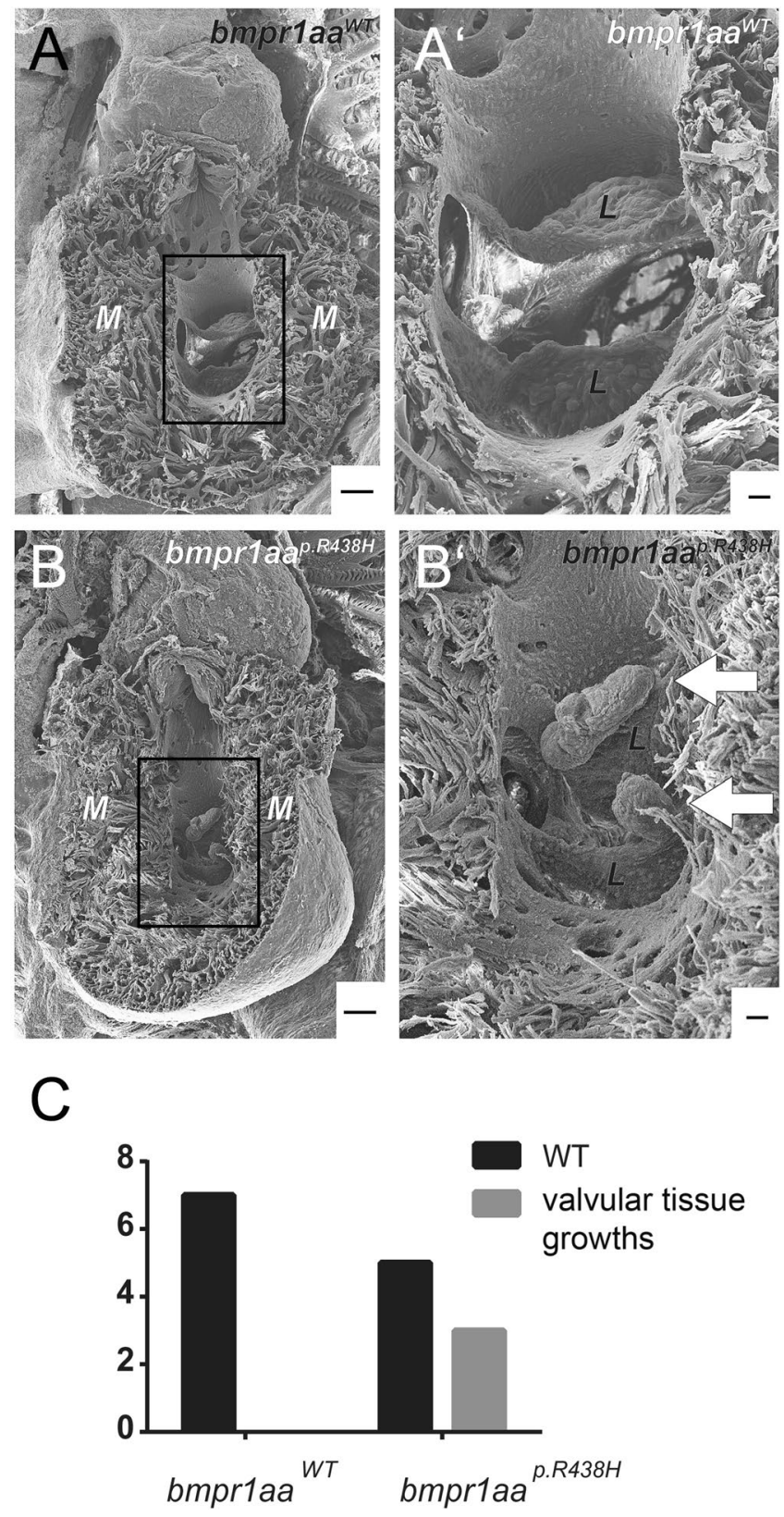

Figure 6. Ectopic valvular tissue growths occur in adult zebrafish overexpressing the bmprlaa ${ }^{p . R 438 H}$ variant within endocardium. (A,B) Electron microscopic images of adult zebrafish hearts with (A) $\mathrm{Tg}(f l i 1 a: G a l 4 F F)^{u b s 3}$; Tg(UAS:bmpr1aa ${ }^{W T}$ IRES_EGFP $)^{m d 65} ; \mathrm{Tg}(7 x T C F-X i a . S i a: N L S-m C h e r r y)^{i a 5}$ or (B) Tg(fli1a:Gal4FF ${ }^{u b s 3}$; Tg(UAS:bmpr1aa ${ }^{p . R 438 H}$ IRES_EGFP $)^{m d 60} ; \operatorname{Tg}(7 x T C F-X i a . S i a: N L S-m C h e r r y)^{i a 5} \cdot \mathrm{M}=$ myocardium. Scale bar $=100 \mu \mathrm{m} .\left(\mathbf{A}^{\prime}, \mathbf{B}^{\prime}\right)$ Magnified view of the atrioventricular valve. $\mathrm{L}=$ valve leaflet. Scale bar $=20 \mu \mathrm{m}$. (A) Adult

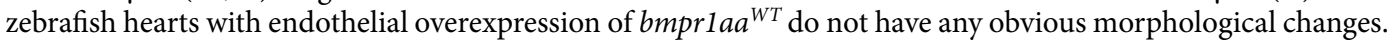
$\left(\mathbf{B}^{\prime}\right) 3$ out of 8 analysed adult zebrafish hearts overexpressing bmpr1aa ${ }^{p \cdot R 438 H}$ have ectopic valvular tissue growth at the atrioventricular valve (arrows). (C) Numbers of zebrafish hearts overexpressing either bmpr 1aa ${ }^{p . R 438 H}$ or bmprlaa ${ }^{W T}$ with ectopic valvular tissue growth. None out of 7 analysed zebrafish hearts overexpressing bmprlaa ${ }^{W T}$ had ectopic valvular tissue growths $(\mathrm{p}=0.200$, fisher's exact test).

with our finding that the injection of human $B M P R 1 A^{p . R 443 H}$ mRNA into zebrafish at the one cell stage complements the knockdown of zebrafish bmprlaa. Currently, we cannot entirely exclude the possibility that the expression of human BMPR1A $A^{p \cdot R 43 H}$ exerts a weak effect on zebrafish embryonic development. The lack of obvious embryonic or cardiac defects upon injection of $B M P R 1 A^{p . R 443 H}$ mRNA into zebrafish provides further evidence for a more complex trait involving the $B M P R 1 A$ mutation together with another modifier in causing cardiac defects. However, as family members are heterozygous carriers, the $B M P R 1 A^{p \cdot R 443 H}$ mutation may also cause a (semi-) dominant effect as assayed in transgenic zebrafish. Although continuous pan-endothelial overexpression of $b m p r 1 a a^{p . R 438 H}$ does not affect embryonic valve development, adult zebrafish hearts are affected. Here, the endothelial overexpression of $\operatorname{bmpr} 1 a a^{p . R 438 H}$ leads to a clear reduction of the AV valve area when compared to the 
overexpression of $b m p r l a a^{W T}$. This effect could be due to a reduced proliferation within the valve leaflets caused by lower Bmprlaa activity.

Strikingly, adult fish overexpressing $b m p r 1 a a^{p . R 438 H}$ in endocardial cells display a severe reduction of $T g$ (7xTCF-Xla.Sia:NLS- $m$ Cherry) ${ }^{i a 5}$ reporter expression, which is indicative of decreased Wnt signalling activity within adult $\mathrm{AV}$ valves. In mice, canonical Wnt signalling acts as a mitogenic trigger of cushion mesenchyme proliferation following endoMT ${ }^{42}$. Indeed, injection of $a p c$ or $d k k-1 \mathrm{mRNA}$, both encoding inhibitors of Wnt/ $\beta$-catenin signalling, induces a complete lack of embryonic endocardial cushion tissue in zebrafish. Correspondingly, zebrafish with a constitutively active Wnt/ $\beta$-catenin signalling have massively expanded atrioventricular endocardial cushions ${ }^{43}$. Similar studies in chicken revealed an increased AVC cell number due to Wnt overexpression $^{45}$. Several lines of evidence suggest that in mice, endoMT within the AVC is accompanied by an upregulation of Wnt/ $\beta$-catenin signalling ${ }^{46}$. The expression of the mesenchymal cell marker $\alpha$ SMA is induced by TGF 32 signalling and TGF $\beta 2$-induced endoMT depends on Wnt/ $\beta$-catenin signalling ${ }^{47}$. Because of the known role of Wnt signalling in cardiac valve development, it is tempting to speculate that the reduced valvular Wnt signalling observed in adult bmpr1aa ${ }^{p . R 438 H}$-overexpressing zebrafish may be directly related to the reduced $\mathrm{AV}$ valve area. Although the exact mechanism of Wnt signalling activation within cardiac valves is currently unknown, it may be a TGF- $\beta$ /Wnt cross-talk, that occurs by reciprocally-regulated ligand production, synergistically regulated shared target genes, or by cytoplasmatic protein interactions ${ }^{48}$. It is unclear why the reduction of the Wnt/ $\beta$-catenin signalling is not apparent in the embryonic heart. Potentially, bmpr1aa ${ }^{p . R 438 H}$-overexpression may only exert a long term and weak effect on this pathway or Wnt/ $\beta$-catenin signalling is more sensitive at later stages of development.

We observed that 3 out of 8 analysed adult zebrafish overexpressing $b m p r 1 a a^{p . R 438 H}$ in endocardial cells develop ectopic valvular tissue mass. The occurrence of these morphological defects in the adult $b m p r 1 a a^{p . R 438 H}$ mutant is not statistically significant due to the small sample size ( $\mathrm{p}=0.200$, fisher's exact test). However, the growth of ectopic valvular tissue mass indicates a tendency of the bmpr1aa ${ }^{p . R 438 H}$ allele to cause defective valve leaflets, something never observed among the control population of animals. Hence, the long-term endocardial

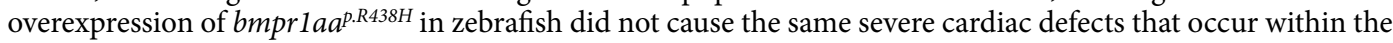
described family ${ }^{25}$. This provides additional evidence for a potentially combinatorial origin of this severe form of CHD. Similar to the human condition that is associated with the BMPR1A $A^{p \cdot R 443 H}$ allele, continuous long-term expression of zebrafish bmpr1aa ${ }^{p \cdot R 438 H}$ alone was not sufficient to cause severe morphological cardiac defects beyond size differences of the cardiac valve leaflets and growths of ectopic valvular tissue mass. This finding lends additional significance to a potentially multigenic combinatorial origin in the aetiology of these inherited CHDs. To elucidate the potential involvement of genetic modifiers within a defined genomic interval on chromosome 1 that co-segregates with the BMPR1 $A^{p \cdot R 443 H}$ mutation in affected family members, a functional characterization of candidate genes is required.

Among the genes homologous to those present within the interval on human chromosome 1, one gene, GIPC $P D Z$ domain containing family, member 2 (gipc2), is a particularly strong candidate that may have a synergistic effect together with $B M P R 1 A^{p . R 443 H}$ in the occurrence of CHDs due to its expression within the zebrafish heart ${ }^{49}$ and its interaction with TGFßR3 in regulating endoMT ${ }^{50}$. This TGFß receptor enhances both BMPR1A and BMPR1B signalling ${ }^{51}$. Such a signalling crosstalk may explain a possible genetic interaction between GIPC2 and $B M P R 1 A^{p . R 443 H}$ in the occurrence of CHDs. In preliminary own knockdown experiments in zebrafish, we have tested several candidate genes from within the $5 \mathrm{Mb}$ interval, including gipc2, nexn, eldt 1 , and fubp 1 , and found that gipc2 showed the strongest phenotype (unpublished own data). However, more substantial functional studies are required to test a potential genetic interaction between any of these candidate genes and bmprlaa during cardiac valve development.

Whole genome sequencing of patient material did not reveal any coding sequence mutations in GIPC2 or any other genes within the linkage interval on chromosome 1 . Nevertheless, changes in regulatory elements or non-coding RNAs may cause alterations in gene expression that were not detected in the patient material. In addition, structural variants could be present in the linkage region, although no copy number variations (CNVs) were detected.

Taken together, we conclude that BMPR1 $\mathrm{A}^{\mathrm{p} \cdot \mathrm{R} 443 \mathrm{H}}$ is a functional receptor variant that leads to a downregulation of Wnt/B-catenin signalling, a reduced AV valve size, and ectopic valvular tissue mass in zebrafish. It is therefore a strong candidate for playing a key role in the development of the reported congenital heart defects. A complex inheritance pattern with an interaction of BMPR1A and modifiers such as GIPC2 in the aetiology of these CHDs seems plausible but needs to be functionally tested in further investigations.

\section{Materials and Methods}

Sequencing. SNP markers were genotyped with the "Genome-Wide Human SNP Array 6.0" by Thermo Fisher Scientific (former Affymetrix). Linkage analysis was performed using the LINKADATAGEN ${ }^{52}$ and MERLIN $^{53}$ software.

Affected individual 8 was exome sequenced at the Helmholtz Zentrum Munich. Family members 13, 15, and 17 were whole-genome sequenced by Complete Genomics (Mountain View, USA) using their proprietary platform. Affected family members 4 and 22 were whole-genome sequenced by Centogene (Rostock, Germany).

We confirm that all methods were carried out in accordance with relevant guidelines and regulations. Moreover, we confirm that all experimental protocols were approved by a named institutional ethics committee (Licence-No.: Neuantrag 2/042 from March $7^{\text {th }}, 2002$, University of Regensburg). Informed consent was obtained from all subjects or from a parent.

Cloning. Plasmids were generated using the Tol2kit and the Gateway system by Invitrogen ${ }^{54}$. Expression clones used for RNA transcription contain a CMV/SP6 promoter. Those used to generate stable transgenic lines 
contain a UAS Promoter. The inserted BMPR1A (human) ORF sequence corresponds to the Ensembl Transcript ID ENST00000372037.7. The bmprlaa (zebrafish) ORF sequence corresponds to nucleotides 456 to 2039 of the Genbank sequence BC115245.1 (http://www.ncbi.nlm.nih.gov/genbank/). Mutagenesis was performed using the QuikChange II XL Site-Directed Mutagenesis Kit. Supplementary Table S3 summarizes the generated expression clones.

Zebrafish strains and maintenance. Zebrafish were kept according to standard laboratory procedures ${ }^{55}$. Handling of zebrafish was done in compliance with German, Berlin and Lower-Saxony state law and carefully monitored by the local authority for animal protection [Landesamt für Gesundheit und Soziales (Berlin, Germany) and Niedersächsisches Landesamt für Verbraucherschutz und Lebensmittelsicherheit (Oldenburg, Germany)]. Breeding and harvesting of fish eggs were authorized by the State Office of consumer protection and food safety Lower-Saxony (Oldenburg, Germany) (Licence-No.: 33.19-42502-04-Demal; 06.01.2016). The generation of transgenic lines was authorized by the State Office of Health and Social Issues (LaGeSo Berlin, Germany) (Licence-No.: Reg0254/12) and by the State Office of consumer protection and food safety Lower-Saxony (Oldenburg, Germany) (Licence-No.: 33.12-42502-04-15/2012). mRNA and MO injections were performed using AB, TüLF, and WIK wildtype strains. To generate transgenic lines, AB, TüLF, and WIK wildtype strains were outcrossed with $\operatorname{Tg}(f l i 1 a: G a l 4 F F)^{u b s 338}$ or $\operatorname{Tg}(k d r l: E G F P)^{s 84340}$. These transgenic lines later were outcrossed with Tg(7xTCF-Xla.Siam:nlsmCherry) ${ }^{\text {ias } 39}$.

mRNA rescue injections. mRNA in vitro transcription was performed using a SP6 polymerase ${ }^{56}$. Zebrafish embryos were injected at 1 -cell stage with $1 \mathrm{nl}$ mRNA $(20 \mathrm{ng} / \mu \mathrm{l})$ und $1 \mathrm{nl}$ morpholino oligo mix [bmprlaa MO (4 mM), bmprlab MO1 (4 mM), bmprlab MO3 (4 mM) 33 (1 mM) in Danieau's Solution]. To prevent cell death by p53 activation due to high MO concentrations, we co-injected p53 MO (tp53 MO4 $)^{57}$. MOs were obtained from Gene Tools and are summarized in Supplementary Table S4. Following the injections, embryos were incubated in eggwater for 24 hours and classified by the severity of their state of dorsalization ${ }^{36}$. Some embryos of each clutch remained uninjected and were used as negative control. Clutches with over $25 \%$ dead embryos at $24 \mathrm{hpf}$ in the negative control were sorted out and not analysed. Only clutches with at least $n \geq 15$ (or $n \geq 10$ in negative control) were analysed. The experiment was performed at least four times with each mRNA variant. The mRNA variants were blinded before injection and unblinded after dorsalization analysis.

As statistical analysis, the mean percentage of dead and C4-malformed embryos were compared between the 4 groups of $\mathrm{MO} / \mathrm{mRNA}$ co-injection and a group with $\mathrm{MO}$ injection only. For this purpose, data was transformed using an arcsine square root transformation. Homoscedasticity and normal distribution were ensured using Levene's test and the Shapiro-Wilk test, respectively. Data was weighted with the square of the number of embryos in the respective clutch. Afterwards the groups were compared using a two-sided student's t-test and the data was corrected using Bonferroni-Holms procedure.

Generation of transgenic lines. The mutation c.G1313A (p.R438H) was introduced in the zebrafish bmprlaa gene and cloned into a Tol 2 vector using the Gateway/Tol2 kit. Constructs were injected into 1-cell stage zebrafish embryos to generate the transgenic lines Tg(UAS:bmpr1aa $\left.{ }^{W T} \_I R E S \_E G F P\right)^{m d 65, m d 67}$ or Tg(UAS:bmpr1aa ${ }^{p . R 438 H}$ IRES_EGFP) ${ }^{m d 60, m d 61, m d 66}$. The founders of these transgenic lines were used to raise stable generations, which were outcrossed with $\mathrm{Tg}(f l i 1 a: G A L 4 F F)^{u b s 3}$ and $\mathrm{Tg}(7 x T C F-X l a . S i a: N L S-m C h e r r y)^{i a 5}$. As some transgenic embryos showed a mosaic endothelial expression pattern of GFP, only embryos with strong expression were selected and raised for a later inspection of AV valve morphology.

Whole-mount in situ hybridization. DIG labeled probe for egfp was generated as previously described ${ }^{58}$. Whole-mount in situ hybridization experiments were performed as previously described ${ }^{59}$. Images were recorded on a stereomicroscope (Leica M165 FC) with an EOS 5 D Mark III (Canon) camera and processed using Adobe Illustrator (Adobe Systems).

PTU treatment and fixation. Tg(fli1a:GAL4FF) ${ }^{u b s 3}$; Tg(UAS:bmprlaa ${ }^{W T}{ }_{-} I R E S$ EGFP)/Tg(UAS:bmpr1aap.R438H_IRES_EGFP); Tg(7xTCF-Xla.Sia:NLS-mCherry)ias embryos were treated with PTU (Sigma) at $24 \mathrm{hpf}$, anaesthetized with 3 -aminobenzoic acid ethyl ester (Tricaine) (Sigma) and fixed in $4 \%$ PFA at $120 \mathrm{dpf}$.

Adult heart extraction. Adult heart extraction was performed according to the published protocol ${ }^{60}$. After extraction of the heart, the atrium was carefully severed from the ventricle exposing the AV valve. Hearts were embedded in $1 \%$ low-melting agarose in glass-bottom dishes with the AV valve facing down and imaged.

Immunohistochemistry. Whole-mount antibody stainings of zebrafish embryos were performed as previously described ${ }^{61}$. The following antibodies were used: mouse anti-ALCAM/Dm-GRASP/ Neurolin (1:200; Developmental Studies Hybridoma Bank). Nuclear stainings were performed using 4',6-diamidino-2-phenylindole (DAPI) (Sigma). We used secondary antibodies conjugated to Alexa-561 or Alexa-647 (Life Technologies) at 1:200.

Preparation and fixation of adult hearts for scanning electron microscopy (SEM). Adult fishes were anaesthesized and transferred to a Petri dish filled with cool Locke's solution $\left(6^{\circ} \mathrm{C}\right)$. The bottom of the Petri dish was covered with a layer of wax. Insect needles were inserted into the mouths, caudal trunks and pectoral fins of the fishes to physically fix them to the bottom of the Petri dish. The pericardial cavity was then opened using microsurgical scissors and the still beating hearts were perfused with Locke's solution (via a micropipette inserted into the sinus venosus) until all visible signs of blood were removed from the heart and bulbus arteriosus. To fix 
hearts in a general dilation, final perfusion was carried out with a calcium-free Locke's solution of $20 \mathrm{mmol} / \mathrm{l}$ manganese chloride ${ }^{62} . \mathrm{MnCl}_{2}$ causes a cardiac arrest in a general dilation by calcium channel blocking. After cardiac arrest, the hearts were externally rinsed with a $25 \%$ solution of glutaraldehyde to achieve a rapid pre-fixation of specimens ${ }^{63}$. Final fixation of the specimens was carried out in a $2 \%$ solution of glutaraldehyde followed by post-fixation in Bouin's solution according to established protocols ${ }^{64}$. The fixed specimens were dehydrated in the usual manner and dried by the critical point method. The dried specimens were mounted on aluminum taps with conducting silver and their ventricles were opened by removal of their ventral myocardial walls using electrolytically sharpened tungsten needles. Specimens were sputter-coated with platinum-palladium (Leica EM ACE 200).

Image acquisition. Confocal images were obtained using a Leica TCS SP8 confocal laser microscope with 20x and 40x magnification for adult and embryonic heart valves, respectively. 3D projections and oblique slices of confocal images were generated using Imaris (Bitplane). Electron microscopy (SEM) images were obtained using a Zeiss Ultra plus field emission scanning electron microscope. Images were processed using Adobe Illustrator CC2015 (Adobe Systems).

Quantification of valve parameters and statistical analysis. Diameter and area of AV valves/ AVCs were measured using Fiji ${ }^{65}$. Embryonic AVC diameter was compared using a two-sided student's t-test. Homoscedasticity and normal distribution were ensured using Levene's test and the Shapiro-Wilk test, respectively. Standard deviation is reported as measure of variability. Adult valve sizes were determined by the measurement of the area bounded by the AV valve annulus. This valve annulus surrounds the edge of the valve leaflets marked by TCF expression and occurs in the confocal images as a black ring. The AV valve measurement technique is shown in detail in Supplementary Fig. S4. The individual measurement data is shown in Supplementary Table S13. For statistical comparison of this adult valve area, analysis of covariance (ANCOVA) was used, which uses linear regression to statistically remove the effects of fish length as covariate before performing a standard ANOVA. Means of AV valve area are reported adjusted for the covariate zebrafish length using ANCOVA.

\section{Data Availability}

Data generated or analysed during zebrafish experiments are included in this published article (and its Supplementary Information files). Sequencing data and all microscopic images are available from the corresponding authors on reasonable request.

\section{References}

1. Lindinger, A., Schwedler, G. \& Hense, H.-W. Prevalence of Congenital Heart Defects in Newborns in Germany: Results of the First Registration Year of the PAN Study (July 2006 to June 2007). Klin. Pädiatr. 222, 321-326 (2010).

2. Hoffman, J. I. Incidence of congenital heart disease: II. Prenatal incidence. Pediatr. Cardiol. 16, 155-165 (1995).

3. Wessels, M. W. \& Willems, P. J. Genetic factors in non-syndromic congenital heart malformations. Clin. Genet. 78, 103-123 (2010).

4. Eisenberg, L. M. \& Markwald, R. R. Molecular regulation of atrioventricular valvuloseptal morphogenesis. Circ. Res. 77, 1-6 (1995).

5. Calkoen, E. E. et al. Atrioventricular septal defect: From embryonic development to long-term follow-up. Int. J. Cardiol. 202, 784-795 (2016).

6. Briggs, L. E., Kakarla, J. \& Wessels, A. The pathogenesis of atrial and atrioventricular septal defects with special emphasis on the role of the dorsal mesenchymal protrusion. Differentiation 84, 117-130 (2012).

7. Kruithof, B. P. T., Duim, S. N., Moerkamp, A. T. \& Goumans, M.-J. TGF $\beta$ and BMP signaling in cardiac cushion formation: Lessons from mice and chicken. Differentiation 84, 89-102 (2012).

8. Paolini, A. \& Abdelilah-Seyfried, S. The mechanobiology of zebrafish cardiac valve leaflet formation. Curr. Opin. Cell Biol. 55, 52-58 (2018).

9. Howe, K. et al. The zebrafish reference genome sequence and its relationship to the human genome. Nature 496, 498-503 (2013).

10. Pestel, J. et al. Real-time 3D visualization of cellular rearrangements during cardiac valve formation. Development 143, 2217-2227 (2016).

11. Steed, E. et al. klf2a couples mechanotransduction and zebrafish valve morphogenesis through fibronectin synthesis. Nat. Commun. 7, 11646 (2016).

12. Kalogirou, S. et al. Intracardiac flow dynamics regulate atrioventricular valve morphogenesis. Cardiovasc. Res. 104, 49-60 (2014).

13. Garside, V. C., Chang, A. C., Karsan, A. \& Hoodless, P. A. Co-ordinating Notch, BMP, and TGF- $\beta$ signaling during heart valve development. Cell. Mol. Life Sci. 70, 2899-2917 (2012).

14. Wang, J., Greene, S. B. \& Martin, J. F. BMP signaling in congenital heart disease: New developments and future directions. Birt. Defects Res. A. Clin. Mol. Teratol. 91, 441-448 (2011).

15. Gaussin, V. et al. Endocardial cushion and myocardial defects after cardiac myocyte-specific conditional deletion of the bone morphogenetic protein receptor ALK3. Proc. Natl. Acad. Sci. USA 99, 2878-2883 (2002).

16. Ma, L. Bmp2 is essential for cardiac cushion epithelial-mesenchymal transition and myocardial patterning. Development 132, 5601-5611 (2005).

17. Breckpot, J. et al. BMPR1A is a candidate gene for congenital heart defects associated with the recurrent $10 \mathrm{q} 22 \mathrm{q} 23$ deletion syndrome. Eur. J. Med. Genet. 55, 12-16 (2012).

18. Menko, F. et al. Variable phenotypes associated with 10q23 microdeletions involving the PTEN and BMPR1A genes. Clin. Genet. 74, 145-154 (2008).

19. Osoegawa, K. et al. Identification of novel candidate gene loci and increased sex chromosome aneuploidy among infants with conotruncal heart defects. Am. J. Med. Genet. A. n/a-n/a https://doi.org/10.1002/ajmg.a.36291 (2013).

20. Septer, S. Aggressive juvenile polyposis in children with chromosome 10q23 deletion. World J. Gastroenterol. 19, 2286 (2013).

21. Zhou, X.-P. et al. Germline Mutations in BMPR1A/ALK3 Cause a Subset of Cases of Juvenile Polyposis Syndrome and of Cowden and Bannayan-Riley-Ruvalcaba Syndromes*. Am. J. Hum. Genet. 69, 704-711 (2001).

22. D'Alessandro, L. C. A. et al. Exome sequencing identifies rare variants in multiple genes in atrioventricular septal defect. Genet. Med. 18, 189-198 (2016).

23. Gaussin, V. Alk3/Bmprla Receptor is Required for Development of the Atrioventricular Canal Into Valves and Annulus Fibrosus. Circ. Res. 97, 219-226 (2005).

24. Muntean, I., Togănel, R. \& Benedek, T. Genetics of Congenital Heart Disease: Past and Present. Biochem. Genet. 55, 105-123 (2017).

25. Schunkert, H. et al. A Large Pedigree With Valvuloseptal Defects. Am. J. Cardiol. 80, 968-970 (1997).

26. Broeckel, U. et al. Suggestive linkage of ebstein's anomaly and concomitant valvuloseptal defects to chromosome 1. Eur. Heart J. 19, $1-688$ (1998). 
27. Kumar, P., Henikoff, S. \& Ng, P. C. Predicting the effects of coding non-synonymous variants on protein function using the SIFT algorithm. Nat. Protoc. 4, 1073-1081 (2009).

28. Ramensky, V. Human non-synonymous SNPs: server and survey. Nucleic Acids Res. 30, 3894-3900 (2002).

29. Schwarz, J. M., Cooper, D. N., Schuelke, M. \& Seelow, D. MutationTaster2: mutation prediction for the deep-sequencing age. Nat. Methods 11, 361-362 (2014).

30. Kircher, M. et al. A general framework for estimating the relative pathogenicity of human genetic variants. Nat. Genet. 46, 310-315 (2014).

31. Quang, D., Chen, Y. \& Xie, X. DANN: a deep learning approach for annotating the pathogenicity of genetic variants. Bioinformatics 31, 761-763 (2015).

32. Ambry Genetics. NM_004329.2(BMPR1A):c.1328G > A (p.Arg443His). ClinVar Registry Available at, https://www.ncbi.nlm.nih. gov/clinvar/variation/231433/\#clinical-assertions (2015).

33. Little, S. C. \& Mullins, M. C. Bone morphogenetic protein heterodimers assemble heteromeric type I receptor complexes to pattern the dorsoventral axis. Nat. Cell Biol. 11, 637-643 (2009).

34. Smith, K. A. et al. Bmp and Nodal Independently Regulate leftyl Expression to Maintain Unilateral Nodal Activity during Left-Right Axis Specification in Zebrafish. PLoS Genet. 7, e1002289 (2011).

35. Howe, J. R. et al. The prevalence of MADH4 and BMPR1A mutations in juvenile polyposis and absence of BMPR2, BMPR1B, and ACVR1 mutations. J. Med. Genet. 41, 484-491 (2004).

36. Mullins, M. C. et al. Genes establishing dorsoventral pattern formation in the zebrafish embryo: the ventral specifying genes. Dev. Camb. Engl. 123, 81-93 (1996).

37. Dietrich, A.-C., Lombardo, V. A., Veerkamp, J., Priller, F. \& Abdelilah-Seyfried, S. Blood flow and Bmp signaling control endocardial chamber morphogenesis. Dev. Cell 30, 367-377 (2014).

38. Herwig, L. et al. Distinct Cellular Mechanisms of Blood Vessel Fusion in the Zebrafish Embryo. Curr. Biol. 21, 1942-1948 (2011).

39. Moro, E. et al. In vivo Wnt signaling tracing through a transgenic biosensor fish reveals novel activity domains. Dev. Biol. 366 , 327-340 (2012)

40. Beis, D. Genetic and cellular analyses of zebrafish atrioventricular cushion and valve development. Development 132, 4193-4204 (2005).

41. Martin, R. T. \& Bartman, T. Analysis of heart valve development in larval zebrafish. Dev. Dyn. Off. Publ. Am. Assoc. Anat. 238, 1796-1802 (2009).

42. Bosada, F. M., Devasthali, V., Jones, K. A. \& Stankunas, K. Wnt/3-catenin signaling enables developmental transitions during valvulogenesis. Dev. Camb. Engl. 143, 1041-1054 (2016).

43. Hurlstone, A. F. L. et al. The Wnt/B-catenin pathway regulates cardiac valve formation. Nature 425, 633-637 (2003).

44. Mishina, Y., Suzuki, A., Ueno, N. \& Behringer, R. R. Bmpr encodes a type I bone morphogenetic protein receptor that is essential for gastrulation during mouse embryogenesis. Genes Dev. 9, 3027-3037 (1995).

45. Person, A. D., Garriock, R. J., Krieg, P. A., Runyan, R. B. \& Klewer, S. E. Frzb modulates Wnt-9a-mediated?-catenin signaling during avian atrioventricular cardiac cushion development. Dev. Biol. 278, 35-48 (2005).

46. Goddard, L. M. et al. Hemodynamic Forces Sculpt Developing Heart Valves through a KLF2-WNT9B Paracrine Signaling Axis. Dev. Cell 43, 274-289.e5 (2017).

47. Liebner, S. et al. B-Catenin is required for endothelial-mesenchymal transformation during heart cushion development in the mouse. J. Cell Biol. 166, 359-367 (2004).

48. Guo, X. \& Wang, X.-F. Signaling cross-talk between TGF-B/BMP and other pathways. Cell Res. 19, 71-88 (2009).

49. Thisse, B. et al. Spatial and temporal expression of the zebrafish genome by large-scale in situ hybridization screening. Methods Cell Biol. 77, 505-519 (2004).

50. Townsend, T. A. et al. Endocardial cell epithelial-mesenchymal transformation requires Type III TGF $\beta$ receptor interaction with GIPC. Cell. Signal. 24, 247-256 (2012).

51. Lee, N. Y., Kirkbride, K. C., Sheu, R. D. \& Blobe, G. C. The Transforming Growth Factor-ß Type III Receptor Mediates Distinct Subcellular Trafficking and Downstream Signaling of Activin-like Kinase (ALK) 3 and ALK6 Receptors. Mol. Biol. Cell 20, 4362-4370 (2009).

52. Bahlo, M. \& Bromhead, C. J. Generating linkage mapping files from Affymetrix SNP chip data. Bioinformatics 25, 1961-1962 (2009).

53. Abecasis, G. R., Cherny, S. S., Cookson, W. O. \& Cardon, L. R. Merlin-rapid analysis of dense genetic maps using sparse gene flow trees. Nat. Genet. 30, 97-101 (2002).

54. Kwan, K. M. et al. The Tol2kit: A multisite gateway-based construction kit for Tol2 transposon transgenesis constructs. Dev. Dyn. 236, 3088-3099 (2007).

55. Westerfield, M. The zebrafish book: A guide for the laboratory use of zebrafish (Danio rerio). (Univ. of Oregon Press, 2007).

56. Melton, D. A. et al. Efficient in vitro synthesis of biologically active RNA and RNA hybridization probes from plasmids containing a bacteriophage SP6 promoter. Nucleic Acids Res. 12, 7035-7056 (1984).

57. Robu, M. E. et al. p53 activation by knockdown technologies. PLoS Genet. 3, e78 (2007).

58. González-Rosa, J. M., Peralta, M. \& Mercader, N. Pan-epicardial lineage tracing reveals that epicardium derived cells give rise to myofibroblasts and perivascular cells during zebrafish heart regeneration. Dev. Biol. 370, 173-186 (2012).

59. Thisse, C. \& Thisse, B. High-resolution in situ hybridization to whole-mount zebrafish embryos. Nat. Protoc. 3, 59-69 (2008).

60. Singleman, C. \& Holtzman, N. G. Heart dissection in larval, juvenile and adult zebrafish, Danio rerio. J. Vis. Exp. JoVE, https://doi. org/10.3791/3165 (2011).

61. Rohr, S. Heart and soul/PRKCi and nagie oko/Mpp5 regulate myocardial coherence and remodeling during cardiac morphogenesis. Development 133, 107-115 (2006).

62. Asami, I. Development of the outflow tract of the rat embryonic heart. (1979).

63. Männer, J. The development of pericardial villi in the chick embryo. Anat. Embryol. (Berl.) 186, 379-385 (1992).

64. Männer, J., Seidl, W. \& Steding, G. Experimental study on the significance of abnormal cardiac looping for the development of cardiovascular anomalies in neural crest-ablated chick embryos. Anat. Embryol. (Berl.) 194, 289-300 (1996).

65. Schindelin, J. et al. Fiji: an open-source platform for biological-image analysis. Nat. Methods 9, 676-682 (2012)

\section{Acknowledgements}

We thank R. Aherrahrou, S. Wrobel, C. Otten, V. Lombardo, A.-C. Dietrich, S. Donat, J. Richter, and S. Kraft for their advice on experimental protocols and for providing fish stocks, reagents or other support. Thanks to A. Tafazzoli and M. Wehle for their help in blinding our studies. We also thank Dr. Reinhard Vonthein (Institut für Biometrie und Statistik, University of Lübeck, Lübeck, Germany) and Dr. Maren Vens (Institut für Medizinische Biometrie und Epidemiologie, Universitätsklinikum Hamburg-Eppendorf, Hamburg, Germany) for their advice on the statistical analysis of our studies. We thank Lisanne Maibücher for proofreading of the manuscript. This work was supported by the Excellence cluster REBIRTH and the DZHK (Deutsches Zentrum für HerzKreislauf-Forschung e.V.). T.D. was supported by the Kaltenbach grant of the Deutsche Herzstiftung e.V., the scholarship "Lübecker Exzellenzmedizin" of the University of Lübeck, and a travel grant of the mobility program 
of the DZHK. M.H. was supported by a fellowship of the Excellence cluster REBIRTH and by a fellowship by the Joachim Herz foundation. S.A.-S. was supported by the Excellence cluster REBIRTH, SFB958, and by Deutsche Forschungsgemeinschaft (DFG) projects SE2016/7-2 and SE2016/10-1.

\section{Author Contributions}

T.D., B.R., I.B., H.S., Z.A., J.E. and S.A.-S. contributed to the conception of the project. B.R. and I.B. collected the family member sequencing data and performed biocomputational analysis. S.A.-S., J.E., T.D., M.H. and Z.A. took part in the experimental design. T.D., M.H., D.D. and J.M. collected the experimental data. T.D., M.H., Z.A., J.E. and S.A.-S. interpreted and analysed the data. T.D., M.H., B.R., H.R., Z.A., H.S., J.E. and S.A.-S. wrote the manuscript and revised the latest version of the manuscript.

\section{Additional Information}

Supplementary information accompanies this paper at https://doi.org/10.1038/s41598-019-39648-7.

Competing Interests: The authors declare no competing interests.

Publisher's note: Springer Nature remains neutral with regard to jurisdictional claims in published maps and institutional affiliations.

(c) (i) Open Access This article is licensed under a Creative Commons Attribution 4.0 International License, which permits use, sharing, adaptation, distribution and reproduction in any medium or format, as long as you give appropriate credit to the original author(s) and the source, provide a link to the Creative Commons license, and indicate if changes were made. The images or other third party material in this article are included in the article's Creative Commons license, unless indicated otherwise in a credit line to the material. If material is not included in the article's Creative Commons license and your intended use is not permitted by statutory regulation or exceeds the permitted use, you will need to obtain permission directly from the copyright holder. To view a copy of this license, visit http://creativecommons.org/licenses/by/4.0/.

(C) The Author(s) 2019 